\title{
ON LATTICE-GAS SPIN MODELS WITH CHIRAL INTERACTIONS
}

\author{
S. Romano ${ }^{1}$, V. Zagrebnov ${ }^{2}$ \\ ${ }^{1}$ Istituto Nazionale per la Fisica della Materia e Dipartimento di Fisica "A. Volta", Universitá di Pavia \\ via A. Bassi 6, I-27100, Pavia, Italy \\ ${ }^{2}$ Université de la Méditerranée and Centre de Physique Théorique, \\ CNRS-Luminy-Case 907 , \\ F-13288 Marseille Cedex 9, France
}

(Received January 13, 2002)

\begin{abstract}
We study here lattice-gas models consisting of two-component classical spins (unit vectors), associated with a three-dimensional lattice, and interacting via a pair potential containing both the scalar product of the two spins, and a chiral (twisting) term; such models can be given magnetic and cholesteric interpretations. We prove that the saturated-lattice version of the model possesses an ordering transition at finite temperature; next we go on to the diluted version, and prove the existence of a chemical potential threshold, above which the model produces long-range orientational order at sufficiently low temperatures. We also comment on extensions of these rigorous results to three-component spins.
\end{abstract}

Key words: lattice spin models, lattice gases, chiral interactions, cholesteric models.

PACS number(s): 05.50.+q, 61.30.-v, 64.70.-p

\section{INTRODUCTION}

Some simple classical lattice spin models, extensively studied in the literature, are defined by pair interaction potentials restricted to nearest neighbours, and of the general form

$$
G=G_{j k}=\epsilon \mathcal{F}(\eta)
$$

here $\epsilon$ denotes a positive quantities setting energy and temperature scales, i. e. $T^{*}=k_{B} T / \epsilon$ (and which can be scaled away from the following equations), and $\eta$ denotes the scalar product between $\nu$-component unit vectors $(\nu=2,3)$ associated with sites of a $d$-dimensional lattice; these interaction models are isotropic in both spin space and lattice space, and some of their commonly studied functional forms are

$$
\begin{aligned}
& \mathcal{F}(\eta)=-\eta \text { (ferromagnetic) } \\
& \mathcal{F}(\eta)=+\eta \text { (antiferromagnetic); } \\
& \mathcal{F}(\eta)=-P_{2}(\eta), \quad \nu=3 \text { (nematogenic lattice model) }
\end{aligned}
$$
in the resulting (continuosly degenerate) ground state, the scalar product of any two spins equals 1 in magnitude, i.e., all particles are aligned in a common direction. In turn, this kind of order may or may not survive at finite temperatures [1]; by now, a number of rigorous results have been worked out, entailing existence or absence (and sometimes type) of phase transitions, depending on lattice dimensionality, number of spin components, symmetry and range of the interaction $[1,2]$. The above spin models are of saturated-lattice (SL) type, i.e., each lattice site is occupied by one and only one spin; their lattice-gas extensions (LG) can also be defined, where each lattice site hosts one spin at most, and site occupation is also controlled by the chemical potential $\mu$.

LG gas extensions of the above models have also been investigated in terms of rigorous statistical mechanical results; it has often been possible to prove that, when the SL model supports an ordering transition at finite temperature, so also do its LG counterparts, at least for sufficiently large chemical potential [3-5].

Moreover, the above lattice spin models have been continuously and vigorously investigated by other techniques as well, e.g., Mean Field treatments, Renormalization Group, high-temperature series expansions of the partition function, simulation, especially in the SL cases.

On the other hand, there also exist other pair potential models containing both a simple polynomial in the scalar product of the two interacting spins, and a "chiral" or "twisting" term, involving their vector product (and to be specified later); this term is usually restricted to nearest neighbours lying on a certain lattice axis ("vertical" nearest neighbours); in some cases, this family of potential models produces a "spiralling" ground state, where spins associated with lattice sites belonging to a given "horizontal" lattice plane are ordered in a common direction, and the the common orientations of neighbouring lattice planes evolve in space in a helical fashion, around the "vertical" lattice direction.

As for physical meaning and physical realizations, let us mention that chiral magnetic models allow for the Dzyaloshinsky-Moriya interaction [6-10]; their counterparts being quadratic in the scalar products have been proposed for cholesteric liquid crystals some twenty-five years ago; they were studied by Mean Field treatment, and, more recently, addressed by computer simulation [11-21].

Let us finally mention that helicoidal order in the ground state can result from appropriate competing antiferromagnetic (or ferro- and antiferromagnetic) interactions, defined by simple scalar products of the spins; however we are not considering such models, also extensively studied in the literature.

The present paper aims at studying some lattice spin models with chiral interactions; we have chosen to start 


\section{S. ROMANO, V. ZAGREBNOV}

with $\nu=2$ component spins for a couple of reasons:

- on the one hand, different potential models become equivalent when $\nu=2$, as explained in detail in the Appendix;

- on the other hand, the case $\nu=2$ allows us to use a well developed apparatus of correlation inequalities [22-29], and thus to faciltate demonstrations.

The scope of the present note is thus twofold:

- on the one hand, we prove existence of an ordering transition at low but finite temperature for (SL) spin models associated with a three-dimensional lattice;

- next we go on to consider their LG extensions, and prove the existence of an ordering transition for sufficiently large chemical potentials

\section{SYMBOLS AND MODELS}

As for other symbols and definitions to be used in the following, we are considering here a classical model, consisting of $\nu$-component unit vectors, associated with a $d$-dimensional lattice $\left\{\mathbf{w}_{k}, k \in \mathbb{Z}^{d}\right\}$ (usually $d=3$ ), and parameterized by the usual polar angles $\left\{\phi_{k}\right\}$ or $\left\{\phi_{k}, \theta_{k}\right\}$; let $\left\{\mathbf{r}_{k}\right\}$ denote their coordinate vectors, let $\left\{\mathbf{e}_{\alpha}, \alpha=1,2,3\right\}$ denote orthonormal basis vectors defined (at least partly) by lattice axes; Cartesian components of the $j$-th unit vector will be denoted by $w_{j}^{\alpha}$; Cartesian coordinates of lattice sites will be noted by the sets of integers $\left(k_{x}, k_{y}, k_{z}\right)$. It will also prove useful to reserve the symbols $\mathbf{u}_{j}$ and $\mathbf{v}_{k}$ for two- and three- component unit vectors, respectively, and to mantain the general one $\mathbf{w}_{k}$ when both cases can be meant; moreover, $\forall k$, let $\mathbf{u}_{k}=\cos \phi_{k} \mathbf{e}_{1}+\sin \phi_{k} \mathbf{e}_{2}$, i. e., all unit vectors $\mathbf{u}_{k}$ are assumed to belong to the "horizontal" $(x, y)$ plane, and to be perpendicular to the "vertical" direction $\mathbf{e}_{3}$. As for three-component unit vectors, in some equations it will prove useful to simplify notation by means of the the symbols $C_{j}=\cos \theta_{j}, S_{j}=\sin \theta_{j}$, etc.. Here and in the following, interaction potentials will be restricted to nearest neighbours $(n n)$; for any pair of nearest-neighbouring sites $j$ and $k$, (and for two-component unit vectors), we also define

$$
\begin{aligned}
& \mathbf{s}_{j k}=\left(\mathbf{r}_{k}-\mathbf{r}_{j}\right), \quad f_{j k}=z_{k}-z_{j}=\mathbf{e}_{3} \cdot \mathbf{s}_{j k}, \\
& \eta_{j k}=\mathbf{u}_{j} \cdot \mathbf{u}_{k}=\cos \left(\phi_{k}-\phi_{j}\right) \\
& \mathbf{t}_{j k}=\mathbf{u}_{j} \wedge \mathbf{u}_{k}=\sin \left(\phi_{k}-\phi_{j}\right) \mathbf{e}_{3}
\end{aligned}
$$

notice that $\mathbf{s}_{j k}$ is a unit vector, coinciding with one of the $\mathbf{e}_{\alpha}$. Consider the three-dimensional lattice $\mathbb{Z}^{d=3}$. Let $\Lambda \subset \mathbb{Z}^{3}$ be a finite prism of the form: $\Lambda=\Lambda_{x y} \times \Lambda_{z}$, where $\Lambda_{x y} \subset \mathbb{Z}^{2}$ and $\Lambda_{z} \subset \mathbb{Z}^{1}$. Let $\Lambda_{x y}^{b}=\left\{\langle i j\rangle \subset \Lambda_{x y}\right\}$ be the set of bonds in $\Lambda_{x y}$, and the same: $\Lambda_{z}^{b}=\left\{\langle s t\rangle \subset \Lambda_{z}\right\}$ in $z$-direction, i.e., $\Lambda^{b}=\Lambda_{x y}^{b} \times \Lambda_{z}^{b}$; the Hamiltonian of the model [16-21] defined by the following nearest-neighbour interaction between two molecules sitting on the bond $\langle j k\rangle$ :

$$
U_{j k}\left(\mathbf{u}_{j}, \mathbf{u}_{k}, \mathbf{s}_{j k}\right)=-J\left[2\left(\mathbf{u}_{j} \cdot \mathbf{u}_{k}\right)^{2}-1\right]-K\left(\mathbf{u}_{j} \cdot \mathbf{u}_{k}\right)\left[\mathbf{s}_{j k} \cdot\left(\mathbf{u}_{j} \wedge \mathbf{u}_{k}\right)\right]
$$

where $J>0$ and $K>0$ denote the coupling constants (strength parameters). Eq. (4) can also be written in a more compact way:

$$
U_{j k}\left(\mathbf{u}_{j}, \mathbf{u}_{k}, \mathbf{s}_{j k}\right)=-J\left(2 \eta_{j k}^{2}-1\right)-K\left[\eta_{j k}\left(\mathbf{s}_{j k} \cdot \mathbf{t}_{j k}\right)\right]
$$

On the other hand, one can make the angular dependence explicit, i.e.,

$$
U_{j k}\left(\mathbf{u}_{j}, \mathbf{u}_{k}, \mathbf{s}_{j k}\right)=\left\{\begin{array}{ll}
-J \cos \left[2\left(\phi_{k}-\phi_{k}\right)\right] \\
-J \cos \left[2\left(\phi_{k}-\phi_{j}\right)\right]-K f_{j k} \sin \left[2\left(\phi_{k}-\phi_{j}\right)\right], \text { hor }
\end{array} ;\right.
$$

where 'hor' and 'ver' denote horizontal (intralayer) and vertical (interlayer) interactions, respectively; in turn, the vertical term can be rewritten

$$
\begin{aligned}
& -J \cos \left[2\left(\phi_{k}-\phi_{j}\right)\right]-K f_{j k} \sin \left[2\left(\phi_{k}-\phi_{j}\right)\right] \\
& =-P \cos \left[2\left(\phi_{k}-\phi_{j}-\tau\right)\right], \\
& P=\sqrt{J^{2}+K^{2}}, \quad \tan (2 \tau)=\frac{K}{J}
\end{aligned}
$$

The Hamitonian of this saturated lattice model is

$$
H_{\Lambda}\left(\left\{\mathbf{u}_{j}\right\}_{j \in \Lambda}\right)=\sum_{\langle j k\rangle \in \Lambda_{b}} U\left(\mathbf{u}_{j}, \mathbf{u}_{k}, \mathbf{s}_{j k}\right)
$$

To make this model in better touch with the fluid nature of liquid crystals we propose its following modification. Associate with each site $j \in \Lambda$ there is a random variable (occupation number) $n_{j}=0,1$. Then we propose a diluted (lattice-gas) version of the model (4): 


$$
\begin{aligned}
& H_{\Lambda}\left(\left\{\mathbf{u}_{j}\right\}_{j \in \Lambda},\left\{n_{j}\right\}_{j \in \Lambda}\right) \\
& =\sum_{\langle j k\rangle \in \Lambda_{b}} U\left(n_{j} \mathbf{u}_{j}, \quad n_{k} \mathbf{u}_{k}, \mathbf{s}_{j k}\right)-\mu \sum_{j \in \Lambda} n_{j} .
\end{aligned}
$$

Here $\mu \in \mathbb{R}^{1}$ is chemical potential of the lattice gas governing the Bernoulli measure for random variables $\left\{n_{j}=0,1\right\}_{j \in \Lambda}$.

In spite of the fact that both of these models are still rather far from a continuous fluid, the lattice-gas generalization has the advantage of allowing for the possible density or pressure dependence of the critical behaviour of liquid crystals.

The aim of the following section is twofold:

- first, we prove that the model (9) manifests a LongRange Order (LRO) parameter for low temperatures; - second, we prove the same for our model (10) for low temperatures and large chemical potentials, i. e., for high densities of the lattice-gas particles.

\section{MAIN RESULTS AND THEIR PROOFS}

In order to establish the existence of LRO in model (4), we introduce the two-spin correlation function

$$
F_{\Lambda, \beta}(k, l)=\left\langle\cos \left[2\left(\phi_{k}-\phi_{l}\right)\right]\right\rangle_{H_{\Lambda}}(\beta), k, l \in \Lambda,
$$

where the right-hand site is the Gibbs expectation value with Hamiltonian (9), for inverse temperature $\beta=\Theta^{-1}$. Since we are dealing with an $O(2)$-symmetric lattice model, we need Reflection Positivity and Infrared Bound techniques [30,31] for the proof of LRO; therefore, some restrictions will be implemented on the model (4), in order to apply [30,31]; possible generalizations will be discussed in Section IV.

First, the interaction (4) itself must be quadratic; it is known [3-5] that for nematics, the appropriate set of variables to express that are real symmetric matrices $\left\{Q_{j}\right\}_{j \in \Lambda}$ :

$$
Q_{j}^{\alpha \gamma}=u_{j}^{\alpha} u_{j}^{\gamma}-(1 / \nu) \delta_{\alpha \gamma}, \alpha, \gamma=1,2, \ldots \nu
$$

associated with unit vectors $\left\{\mathbf{u}_{j}\right\}_{j \in \Lambda}$ for $\nu=2$, see [3]. Then

$$
U^{\mathrm{h} \circ \mathrm{r}}\left(\phi_{j}, \phi_{k}\right)=-2 J \operatorname{Tr}\left(Q_{j} \cdot Q_{k}\right), j_{z}=k_{z}
$$

and

$$
\begin{array}{r}
U^{\text {ver }}\left(\phi_{s}, \phi_{t}\right)=-2 P \operatorname{Tr}\left(Q_{s} \mathcal{R}_{t_{z}-s_{z}} Q_{t} \mathcal{R}_{t_{z}-s_{z}}^{*}\right) \\
-2 P \operatorname{Tr}\left(\mathcal{R}_{s_{z}} Q_{s} \mathcal{R}_{s_{z}}^{*} \cdot \mathcal{R}_{t_{z}} Q_{t} \mathcal{R}_{t_{z}}^{*}\right)
\end{array}
$$

for $\langle s t\rangle \subset \Lambda_{z}^{l}$. Here the orthogonal transformations $\left\{\mathcal{R}_{t,}, t \in \Lambda\right\}$ are implemented by uniform relative rotations of molecules in two adjacent layers $\Lambda_{x y}^{s}, \Lambda_{x y}^{t}$, $\left|t_{z}-s_{z}\right|=1$, by the helical wave-vector $q$, based on the treatment in Ref. [10]

$$
\begin{aligned}
u_{t} & =\mathcal{R}_{t_{z}-s_{z}} u_{s}=\mathrm{e}_{1} \cos \left[\phi_{s}+\left(t_{z}-s_{z}\right) q\right] \\
& +\mathrm{e}_{2} \sin \left[\phi_{s}+\left(t_{z}-s_{z}\right) q\right] .
\end{aligned}
$$

Upon changing matrix variables to

$$
\tilde{Q}_{k}=\mathcal{R}_{k_{z}} Q_{k} \mathcal{R}_{k_{z}}^{*}, k \in \Lambda,
$$

we get for interactions (13) and (14)

$$
\tilde{U}^{\mathrm{hor}}\left(\psi_{j}, \psi_{k}\right)=-2 J \operatorname{Tr}\left(\tilde{Q}_{j} \tilde{Q}_{k}\right), j_{z}=k_{z}
$$

and

$$
\tilde{U}^{\text {ver }}\left(\psi_{s}, \psi_{t}\right)=-2 P \operatorname{Tr}\left(\tilde{Q}_{s} \tilde{Q}_{t}\right)
$$

where $\psi_{k}=\phi_{k}-k_{z} q$ and $\langle s t\rangle \subset \Lambda_{z}^{l}$. Therefore, after the helical transformation (Eq. (15) and (16), the interaction stays quadratic and purely nematic.

The second restriction applicable $[30,31]$ is to a nearest-neigbhour interaction (or to long-ranged one of appropriate type), and which is attractive. Both aspects are present in the original interaction (4) as accepted in the literature, e.g., Refs. [11-14]; we do not insist here on these restrictions, see Section IV.

The third restriction concerns periodic boundary conditions on $\partial \Lambda$, and poses no problem in our case.

After assemblying all the necessary elements [30], [31], we are now able to state the first result.

\section{Theorem 1}

There exists a temperature

$$
\Theta_{0}:=8 J(m-1) /\left[m^{2}(m+1) D\right], m=2,
$$

where

$$
\begin{aligned}
& D:=(2 \pi)^{-3} \int_{\mathcal{B}} d^{3} \mathbf{p}\left[\sum_{k=1}^{k=3}\left(1-\cos p_{k}\right)\right]^{-1}, \\
& \mathcal{B}=[-\pi,+\pi]^{3}
\end{aligned}
$$

and such that, for all $\Theta<\Theta_{0}$, the model defined by Eq. (9) manifests a non-zero translationally invariant LRO in $x y$-planes, as well as helical LRO in the $z$-direction:

$$
\left\langle Q_{j}\right\rangle\left(\beta>\beta_{0}\right)=\mathcal{R}_{j_{z}}^{*}\left\langle\tilde{Q}_{j=0}\right\rangle\left(\beta>\beta_{0}\right) \mathcal{R}_{j_{z}}, j \in \mathbb{Z}^{3} .
$$

\section{$\underline{\text { Proof }}$}

Notice that $\operatorname{Tr} Q_{j}=0$, i.e., $\operatorname{Tr}\left\langle Q_{j}\right\rangle_{\tilde{H}_{\Lambda}}=0$, and by the $O(2)$ invariance of the system one gets $\left\langle\tilde{Q}_{j}\right\rangle_{\tilde{H}_{\Lambda}}=0$. Therefore, in oder to establish the existence of LRO one 
has to prove that

$$
\begin{aligned}
& \lim _{|k-l| \rightarrow \infty} \lim _{\Lambda \uparrow \mathbb{Z}^{3}} \tilde{F}_{\Lambda, \beta}(k, l) \\
= & \lim _{|k-l| \rightarrow \infty} \lim _{\Lambda \uparrow \mathbb{Z}^{3}}\left\langle\cos 2\left(\psi_{k}-\psi_{l}\right)\right\rangle_{\tilde{H}_{\Lambda}}(\beta) \\
= & \lim _{|k-l| \rightarrow \infty} \lim _{\Lambda \uparrow \mathbb{Z}^{3}} 2 \operatorname{Tr}\left\langle\tilde{Q}_{k} \tilde{Q}_{l}\right\rangle_{\tilde{H}_{\Lambda}}(\beta) \neq 0
\end{aligned}
$$

for low temperatures. This statement in due to the techniques of $[30,31]$, as was demonstrated in [3]. Since the interaction in $\hat{H}_{\Lambda}$ is anisotropic, we get the estimate of temperature $\Theta_{0}$ (Eq. (19)) as developed and discussed in Refs. [5,32], with reference to the smallest coupling $J$. The statement (21) is immediate from the helical transformation (Eqns. (16) and (16)).

Next we state and prove a similar result for the diluted (lattice-gas) version of the model (10).

\section{Theorem 2}

There exists a chemical potential $\mu_{0}$ such that, for every $\mu>\mu_{0}$ there is a $\Theta_{0}(\mu)$, such that, for all $\Theta<\Theta_{0}(\mu)$ the model (10) manifests a non-zero translation-invariant LRO in the $x y$-planes, and helicoidal LRO in the $z$ direction.

$$
\begin{aligned}
& \left\langle n_{j} Q_{j}\right\rangle\left(\beta>\beta_{0}(\mu), \mu>\mu_{0}\right) \\
& =\mathcal{R}_{j_{z}}^{*}\left\langle n_{j} \tilde{Q}_{j_{z}=0}\right\rangle\left(\beta>\beta_{0}(\mu), \mu>\mu_{0}\right) \mathcal{R}_{j_{z}}, j \in \mathbb{Z}^{3} .
\end{aligned}
$$

\section{$\underline{\text { Proof }}$}

Notice that, after carrying out the helical transformation (Eqns. (16) and (16)) the model (4) reduces to a nematic lattice-gas model with a nearest-neighbour attractive (ferromagnetic) coupling, known since [3]; by virtue of the reasoning in [5], one obtains that $\left\langle n_{j} \tilde{Q}_{j}\right\rangle(\beta, \mu) \neq 0$ is ensured by

$$
L(\beta, \mu)-I_{d=3}(\beta)>0
$$

Here (for some $\xi>0$ and $c>0$ )

$$
\begin{aligned}
L(\beta, \mu) & =(1 / 2)\{1-(1 / \xi) \\
& \times \exp [-\beta(c(4 J+2 P+\mu)]\}
\end{aligned}
$$

and

$$
\begin{aligned}
I_{d=3}(\beta) & =\frac{\Theta}{(2 \pi)^{3}} \int_{\mathcal{B}}\left[J \sum_{k=1}^{k=2}\left(1-\cos p_{k}\right)\right. \\
& \left.+P\left(1-\cos p_{3}\right)\right]^{-1} .
\end{aligned}
$$

From these equations it is clear that, for

$$
\mu>\mu_{0}:=-c\left(4 J+2 \sqrt{J^{2}+K^{2}}\right),
$$

there exists a $\beta_{0}(\mu)$ such that

$$
L\left(\beta_{0}(\mu), \mu\right)=I_{d=3}\left(\beta_{0}(\mu)\right)
$$

and that the inequality (24) is ensured in the domain

$$
\left\{\mu, \Theta: \mu>\mu_{0}, \Theta<\left(\beta_{0}(\mu)\right)^{-1}\right\}
$$

Finally, Eq. (23) follows from the helical transformation.

\section{COMMENTS}

\section{Subsection 4.1}

We first comment on the conditions for the proof of Theorem 1.

a) The model (9) manifests helical LRO at low temperatures, with a temperature-independent wave vector $q$, and this does not seem to be very realistic from the physical point of view. In order to make the helical wavevector temperature-dependent, one has to perturb the interaction $U^{\text {hor }}$ or $U^{\text {ver }}$ by some "anharmonic" terms, which, a priori, does not allow to use the techniques from Refs. [30,31]. One possibility used in Mean-Field and simulation work (see, e. g., Ref. [18]) is to add a quartic term to $U^{\text {hor }}$

$$
\tilde{U}^{\mathrm{hor}}\left(\phi_{j}, \phi_{k}\right)=U^{\mathrm{hor}}\left(\phi_{j}, \phi_{k}\right)-J_{4}\left[\cos \left(\phi_{j}-\phi_{k}\right)\right]^{4}
$$

\section{Corollary 1.1}

For $J_{4}>0$ there exists a temperature $\hat{\Theta}_{0}$ such that, for all $\Theta<\hat{\Theta}_{0}$ a perturbed model with interaction $\hat{U}^{\text {hor }}+U^{\text {ver }}$ manifests LRO similar to that in Theorem 1.

\section{$\underline{\text { Proof }}$}

The quartic perturbation makes the interaction $\hat{U}^{\text {hor }}+$ $U^{\text {ver }}$ non-quadratic, and thus it harms the technique based on Refs. [30,31]. On the other hand, since $J_{4} \geq 0$ (ferromagnetic perturbation), one can use the Ginibre inequalities [24] for the ferromagnetic plane-rotator model with Hamiltonian $\hat{H}_{\Lambda}$ to estimate the corresponding twospin correlation function from below:

$$
\left\langle\cos \left[2\left(\phi_{k}-\phi_{l}\right)\right]\right\rangle_{\hat{H}_{\Lambda}}(\beta) \geq F_{\Lambda, \beta}(k, l)
$$

This inequality, together with the statement (21) in Theorem 1, finishes the proof.

\section{Remark}

One could equally well consider adding a similar quartic term to $U^{\text {ver }}$, or add quartic terms to both $U^{\text {hor }}$ and $U^{\text {ver }}$. 
b) Again, by Ginibre inequalities [24], we can relax the nearest-neighbour interaction in (4) to any ferromagnetic (long-range) interaction $U_{\text {ferro }}^{\text {hor }}\left(\phi_{j}, \phi_{k}\right)$ or $U_{\text {ferro }}^{\text {ver }}\left(\phi_{j}, \phi_{k}\right)$. If $H_{\Lambda}^{\mathrm{nn}}$ corresponds to the nearest-neighbour restriction of the longer-range interactions, then

$$
\left\langle\cos \left[2\left(\phi_{j}-\phi_{k}\right)\right]\right\rangle_{H_{\Lambda}^{\mathrm{ferro}}} \geq\left\langle\cos \left[2\left(\phi_{j}-\phi_{k}\right)\right]\right\rangle_{H_{\Lambda}^{\mathrm{nn}}}
$$

Now by Theorem 1 one gets LRO in the system with $H_{\Lambda}^{\text {ferro }}$ for sufficiently low temperature.

\section{Remark 3.1}

In both previous cases a) and b), one proves the existence of LRO, but can not control the value of the helical wave vector. c) The ferromagnetic character of the nearestneigbhbour interaction can be relaxed; actually one can equally well consider the antiferromagnetic version of this model (spin-flip symmetry). Here $\pi / 2$ rotations of the internal frame systems on the odd (or even) sublattices of $\mathbb{Z}^{3}$ result in the change of sign for the coupling constants, and this change of variables maps the $O(2)$ antinematic model to the ferromagnetic chiral model (4), so that Theorem 1 is again applicable.

d) Instead of planar rotators $\left\{\mathbf{u}_{j}, j \in \mathbb{Z}^{3}, \mathbf{u}_{j} \in\right.$ $\left.\mathbb{S}^{1}\right\}$, let us now consider three-component unit vectors $\left\{\mathbf{v}_{j}, j \in \mathbb{Z}^{3}, \mathbf{v}_{j} \in \mathbb{S}^{2}\right\}$; We address first the extreme anisotropic case where only $x$ - and $y$-spin components are involved in the interaction ( $x y$ model): more precisely, we consider here the model

$$
V_{x y}^{\mathrm{hor}}\left(\theta_{j}, \phi_{j}, \theta_{k}, \phi_{k}\right)=-J\left(\sin \theta_{j} \sin \theta_{k}\right) \cos \left[2\left(\phi_{j}-\phi_{k}\right)\right]
$$

and

$$
V_{x y}^{\text {ver }}\left(\theta_{j}, \phi_{j}, \theta_{k}, \phi_{k}\right)=-\sqrt{J^{2}+K^{2}}\left(\sin \theta_{j} \sin \theta_{k}\right) \cos \left[2\left(\phi_{j}-\phi_{k}-q\right)\right] .
$$

Then, upon applying Wells' inequality [25-29], one readily gets the estimate

$$
\left\langle\cos \left[2\left(\phi_{k}-\phi_{l}\right)\right]\right\rangle_{H_{\Lambda}}(\epsilon \beta) \leq\left\langle\cos \left[2\left(\phi_{k}-\phi_{l}\right)\right]\right\rangle_{H_{\Lambda}^{x y}}(\beta) \leq\left\langle\cos \left[2\left(\phi_{k}-\phi_{l}\right)\right]\right\rangle_{H_{\Lambda}}(\beta)
$$

where $\epsilon<1$ is a positive constant, independent of $\beta$. Together with Theorem 1, this estimate entails that the $x y$ model exhibits $L R O$ for $\Theta<\epsilon \Theta_{0}$. In the Appendix we show that a similar inequality holds for more general interactions.

\section{Subsection 4.2}

We present here some remarks concerning the latticegas model (4) and Theorem 2.

a) Due to the results in Ref. [33], we can tell something about how the nematic/helical LRO appears, and about its correlation with the behaviour of the mean occupation number (particle density) $\rho(\beta, \mu)=\left\langle n_{j}\right\rangle(\beta, \mu)$.

- We first note that, by the chiral transformation (Eqns. (16) and (16)), the chiral nematic can be mapped to the non-chiral one:

- we next note that, by the simple change of variables $\left\{2 \psi_{j}=\tilde{\psi}_{j}, j \in \mathbb{Z}^{3}\right\}$, the nematic model becomes equivalent to a lattice-gas model involving ferromagnetic $O(2)$-symmetric plane rotators, and for which it has been possible to prove that, for sufficiently large $\beta>\beta^{*}$, there exists a line $\mu_{t}(\beta)$, across which the system exhibits a first-order transition, involving discontinuities in both magnetization $\langle\mathbf{u}\rangle(\beta, \mu)$ and density $\rho(\beta, \mu)$; therefore, the same behaviour ensues for the present model (4).

\section{ACKNOWLEDGEMENTS}

This paper originated during VAZ's stay at the Physics Department of Pavia University, funded by the Italian Consiglio Nazionale delle Ricerche (CNR), through a short-term visiting fellowship (STM scheme); we acknowledge financial support and scientific hospitality, respectively.

\section{APPENDIX}

Equivalence of potential models

Consider the integrals

$$
\begin{gathered}
\mathcal{I}_{1}=\int_{0}^{2 \pi} \Delta(\cos t, \sin t) d t, \\
\mathcal{I}_{m}=\int_{0}^{2 \pi} \Delta(\cos m t, \sin m t) d t
\end{gathered}
$$

where $\Delta$ is an arbitray integrable function, and $m \neq 0$ is an arbitrary integer; by a change of variable ( $t$ to $m t$ ) one obtains $\mathcal{I}_{m}=\mathcal{I}_{1}, \forall m$ [34,35]; moreover [34,35], on the basis of the trigonometric identity 


$$
\sum_{k=1}^{k=M} \exp \left( \pm \frac{2 \pi k}{M} i\right)=0, M \geq 2
$$

it easy to check that

$$
\int_{0}^{2 \pi} \exp ( \pm i S t) \Delta(\cos m t, \sin m t) d t=0
$$

when $S \neq 0$ is an integer number but not a multiple of $m$; on the other hand, when $S$ is a multiple of $m$, say $S=\sigma m$, one is again reduced to Eq. (36), so that the value of the integral depends on $\sigma$ but not on $m$.

Now consider two arbitrary real numbers $a$ and $b$, and the interaction potential(s)

$$
U_{m}=U_{j k ; m}= \begin{cases}a \cos \left[m\left(\phi_{k}-\phi_{k}\right)\right] & , \text { hor } \\ b \cos \left[m \cos \left(\phi_{k}-\phi_{j}\right)\right] & \text {, ver }\end{cases}
$$

one can easily check that, for assigned values of $a$ and $b$, all potentials $U_{j k ; m}$ lead to the same partition function (hence thermodynamic properties), and essentially to the same structural properties; the choice $m=1$ defines magnetic models, and the choice $m=2$ may lead to nematogenic one. Moreover, let $m=1$, and consider the four potential models defined by common values of $|a|$, $|b|$, and only differing in the sign of the named parameters; since the lattice is bipartite, spin-flip symmetry entails that, in the absence of external fields, the four models again produce the same partition function, and esentially the same structural properties, i.e., potential models only differing on the signs of the named parameters produce correlation functions related by known sign factors. Notice that spin-flip symmetry holds for threecomponent spins as well, and in general, for a bipartite lattice and nearest-neighbour interactions defined by an arbitrary odd function of spin components. Thus, Eq. (39) can be reduced to the "generalized ferromagnetic" (GFM) case

$$
\begin{aligned}
& V_{j k ; m}=\left\{\begin{array}{ll}
-A \cos \left[m\left(\phi_{k}-\phi_{k}\right)\right] & \text { hor } \\
-B \cos \left[m \cos \left(\phi_{k}-\phi_{j}\right)\right] & \text {, ver }
\end{array},\right. \\
& A>0, B>0
\end{aligned}
$$

where the choice $m=1$ defines the strict ferromagnetic (FM) interpretation, whereas the choice $m=2$ defines a nematogenic lattice model.

Next we consider the potential model(s)

$$
W_{j k ; m}= \begin{cases}a \cos \left[m\left(\phi_{k}-\phi_{k}\right)\right] & , \text { hor } \\ b \cos \left[m\left(\phi_{k}-\phi_{j}\right)\right]+c f_{j k} \sin \left[m\left(\phi_{k}-\phi_{j}\right)\right] & , \text { ver }\end{cases}
$$

where the vertical term can be rewritten

$$
\begin{aligned}
& b \cos \left[m\left(\phi_{k}-\phi_{j}\right)\right]+c f_{j k} \sin \left[m\left(\phi_{k}-\phi_{j}\right)\right]=\rho \cos \left[m\left(\phi_{j}-\phi_{k}-\tau\right)\right], \\
& \rho=\sqrt{b^{2}+c^{2}}, \tan (m \tau)=\frac{c}{b}
\end{aligned}
$$

By the same argument as above, one recognizes the equivalence of potential models defined by the same values of $a, b$ and $c$, but different values of $m$. In the following, we shall be considering and comparing two potentail models defined by

$$
\begin{aligned}
& W^{\prime}=a^{\prime} \eta_{j k}+b^{\prime} f_{j k}\left(\mathbf{e}_{3} \cdot \mathbf{t}_{j k}\right) \\
& W^{\prime \prime}=a^{\prime \prime}\left(2 \eta_{j k}^{2}-1\right)+b^{\prime \prime} \eta_{j k}\left(\mathbf{s}_{j k} \cdot \mathbf{t}_{j k}\right) .
\end{aligned}
$$

Upon expanding the formulae, and recalling the previous analysis (especially Eqs. (36) to (38)), one can recognize that the two potential models $W^{\prime}$ and $W^{\prime \prime}$ produce the same thermodynamic and structural properties provided that

$$
a^{\prime \prime}=a^{\prime}, b^{\prime \prime} / 2=b^{\prime} .
$$

Let us now restrict our attention to Eq. (42), and let $\Xi$ denote an arbitrary number; each polar angle $\phi_{k}$ can be identically decomposed

$$
\phi_{k}=\Xi z_{k}+\psi_{k}
$$

so that

$$
\cos \left(\phi_{j}-\phi_{k}\right)= \begin{cases}\cos \left(\psi_{k}-\psi_{j}\right) & , \text { hor } \\ \cos \left[\left(z_{k}-z_{j}\right) \Xi+\left(\psi_{k}-\psi_{j}\right)\right] & , \text { ver }\end{cases}
$$


After allowing for Eqs. (43) and (44), and choosing $\Xi=\tau$, Eq. (42) becomes

$$
W_{j k}^{\prime}=\left\{\begin{array}{ll}
a^{\prime} \cos \left(\psi_{k}-\psi_{j}\right) & , \text { hor } \\
\rho^{\prime} \cos \left(\psi_{k}-\psi_{j}\right) & , \text { ver }
\end{array}, \quad \rho^{\prime}=\sqrt{\left(a^{\prime}\right)^{2}+\left(b^{\prime}\right)^{2}}\right.
$$

and a similar transformation can be applied on Eq. (46); this approach is based on Ref. [10]. With appropriate signs of the parameters, Eqs. (42) produces a helical ground state where each layer is completely ordered ferromagnetically, and the common orientations of two consecutive layers are rotated by an angle $\tau$; on the other hand, the previous discussion shows how this twist can be transformed away; since the transformation is temperature independent, the pitch is also independent of temperature, if order survives at finite temperature (i.e., when $d \geq 3$ ).

The interaction term containing the vector product $\mathbf{t}_{j k}$ in Eq. (42) was proposed some thirty-five years ago by Dzaloshinsky [6] and Moriya [7], as a model explaining the phenomenon of weak ferromagnetism in systems with predominant antiferromagnetic interactions [8-10]; in microscopic terms, it originates from spin-orbit interactions; extensions to three-component spins are also known, and there also the ground-state twist can be transformed away. This model has been extensively investigated, both for classical and quantum spins (see, e.g., Refs. [36-40] for a few papers published after 1990); exact solutions are known when $d=1$ [41-43].

\section{Some remarks on chiral nematic models}

Nematogenic molecules are usually neither rigid nor cylindrically symmetric, and often possess appreciable dipole moments, yet the resulting thermotropic nematic phases are apolar and usually possess uniaxial symmetry; therefore, the simplifying assumption of uniaxial molecular symmetry $\left(C_{\infty v}\right.$, or, more frequently $\left.D_{\infty h}\right)$ has been extensively used in theoretical treatments; moreover, to a reasonable approximation, the existence or absence of long-range orientational order marks the one difference between a nematic phase and the isotropic liquid to which it transits at higher temperature; this has suggested the additional simplification of nematogenic lattice models, also extensively studied in the literature. In cholesteric liquid crystals, there exists locally nematic orientational order, and this locally preferred orientation spirals in space, around an axis perpendicular to it; the pitch of the helix is in general temperature-dependent, and in some cases it can even change sign with temperature.

On the other hand, the corresponding term in Eq. (46) was proposed and discussed some twenty-five years ago, especially by van der Meer et al. [11-14]; the interaction model is based on a perturbation treatment of intermolecular interactions, and on further assumptions, as discussed in the original papers, for example:

- on a multipolar expansion of Coulombian interac- tions between the two particles, and on the neglect of permanent multipolar terms; notice that short-range repulsion between the two molecules is not explictly included, and is often allowed for by constraining molecules on a lattice;

- on the assumption of an effective cylindrical symmetry of the interacting molecules; After additional simplifications, the following potential model is defined in the named papers:

$$
\begin{aligned}
& W_{j k}=W\left(\mathbf{w}_{j}, \mathbf{w}_{k}, \mathbf{x}_{j}-\mathbf{x}_{k}\right) \\
& =-J \eta_{j k}^{2}-K \eta_{j k}\left(\mathbf{r}_{j k} \cdot \mathbf{t}_{j k}\right)-L \eta_{j k}^{4}-M \eta_{j k}^{3}\left(\mathbf{r}_{j k} \cdot \mathbf{t}_{j k}\right)
\end{aligned}
$$

where $\mathbf{w}_{j}$ and $\mathbf{w}_{k}$ denote 2 - or 3 -component unit vectors defining molecular orientations, $\mathbf{x}_{j}$ and $\mathbf{x}_{k}$ denote their continuous centre-of-mass coordinates,

$$
\begin{aligned}
& \mathbf{R}_{j k}=\mathbf{x}_{j}-\mathbf{x}_{k}, \quad r_{j k}=\frac{\mathbf{R}_{j k}}{\left|\mathbf{R}_{j} k\right|} \\
& \eta_{j k}=\mathbf{w}_{j} \cdot \mathbf{w}_{k}, \quad \mathbf{t}_{j k}=\mathbf{w}_{j} \wedge \mathbf{w}_{k} .
\end{aligned}
$$

In general the coefficients $J, K, L, M$ are functions of the distance between the two centers of mass [11-14]; on the other hand, when a lattice model with nearest-neighbour interaction is considered, the named coefficients just become adjustable parameters. Notice also that $J>0$, and that signs of $K$ and $M$ can be taken as positive without loss of generality. The above equation was derived for three-component unit vectors [12-14]; on the other hand, a further simplifications has often been proposed and used, i.e., the unit vectors have been constrained to two components, on a plane orthogonal to the axis of the helix. The resulting models have been studied by Mean Field or Two-Site Cluster treatment, as well as by Monte Carlo simulation [11,15-21]. The Authors of some recent papers have tried to explicitly allow for molecular biaxiality [44-47].

Let us finally consider the three-component counterpart of $W^{\prime \prime}$, i.e.,

$$
\begin{aligned}
& W^{\prime \prime \prime}=-a\left[(3 / 2)\left(\mathbf{v}_{j} \cdot \mathbf{v}_{k}\right)^{2}-(1 / 2)\right] \\
& +b\left(\mathbf{v}_{j} \cdot \mathbf{v}_{k}\right)\left(z_{j}-z_{k}\right)\left[\mathrm{e}_{3} \cdot\left(\mathbf{v}_{j} \wedge \mathbf{v}_{k}\right)\right], \quad a>0
\end{aligned}
$$

where the $b$ term does not act among horizontal neighbours, and the vertical interaction reads: 


$$
\begin{aligned}
& W_{\text {ver }}^{\prime \prime \prime}=-a(3 / 2)\left[\left(C_{j} C_{k}\right)^{2}\right. \\
& \left.+2 S_{j} C_{j} S_{k} C_{k} \cos \left(\phi_{k}-\phi_{j}\right)+\left(S_{j} S_{K}\right)^{2} \cos \left(\phi_{k}-\phi_{j}\right)^{2}\right] \\
& +a / 2+b\left(z_{k}-z_{j}\right)\left(C_{j} C_{k}+S_{j} S_{k} \cos \left(\phi_{k}-\phi_{j}\right)\right) \\
& \times S_{j} S_{k} \sin \left(\phi_{k}-\phi_{j}\right)
\end{aligned}
$$

i. e.,

$$
\begin{aligned}
& W^{\prime \prime \prime}=C_{j} S_{j} C_{k} S_{k}\left[3 a \cos \left(\phi_{k}-\phi_{j}\right)\right. \\
& \left.+b\left(z_{k}-z_{j}\right) \sin \left(\phi_{k}-\phi_{j}\right)\right] \\
& -\left(S_{j} S_{k}\right)^{2}\left\{(3 / 2) a(1 / 2)\left[1+\cos \left(2\left(\phi_{k}-\phi_{j}\right)\right)\right]\right. \\
& \left.+(1 / 2) b\left(z_{k}-z_{j}\right) \sin \left[2\left(\phi_{k}-\phi_{j}\right)\right]\right\}+a / 2
\end{aligned}
$$

$$
W^{\prime \prime \prime}=-a P_{2}\left(C_{j}\right) P_{2}\left(C_{k}\right)
$$$$
-\sqrt{(3 a)^{2}+b^{2}} C_{j} S_{j} C_{k} S_{k} \cos \left(\phi_{k}-\phi_{j}-\tau\right)
$$

$$
-\sqrt{(3 a / 4)^{2}+(b / 2)^{2}}\left(S_{j} S_{k}\right)^{2} \cos \left[2\left(\phi_{k}-\phi_{j}-\tau^{\prime}\right)\right],
$$

where

$$
\tan \tau=\frac{b}{3 a}, \quad \tan \left(2 \tau^{\prime}\right)=2 \frac{b}{3 a}
$$

There seems to be no general way of simplifying these equations; on the other hand, in the limit $0<|b| / a \ll 1$, one can approximate $\tan \left(2 \tau^{\prime}\right)=\tan (2 \tau)$ so that the interaction potential becomes

$$
\begin{aligned}
& W^{\prime \prime \prime}=-a P_{2}\left(C_{j}\right) P_{2}\left(C_{k}\right) \\
& -\sqrt{(3 a)^{2}+b^{2}}\left(C_{j} S_{j}\right)\left(C_{k} S_{k}\right) \cos \left(\phi_{k}-\phi_{j}-\tau\right) \\
& -\sqrt{(3 a / 4)^{2}+(b / 2)^{2}}\left(S_{j} S_{k}\right)^{2} \cos \left[2\left(\phi_{k}-\phi_{j}-\tau\right)\right] ;
\end{aligned}
$$

one recognizes at this stage that the twisting term can be transformed away, along the previous lines; moreover, reflection positivity in the interaction still holds, and one can again conclude the existence of an orientational ordering transition at low but finite temperature; in the named limit, the chiral wave vector becomes temperature-independent; this was also noted in the Mean Field treatment of Ref. [12].

\section{Bounds on transition temperatures}

To be specific, let us consider the layered FM model defined by

$$
\begin{aligned}
& V_{j k ; m}=\left\{\begin{array}{ll}
-A \cos \left(\phi_{k}-\phi_{j}\right) & , \text { hor } \\
-B \cos \left(\phi_{k}-\phi_{j}\right) & , \text { ver }
\end{array},\right. \\
& A>0, B>0
\end{aligned}
$$

extensively studied in the literature; the model defined by $A=B=1$ is rigorously known to possess an ordering transition, whose transition temperature may be conservatively estimated to be $\Theta_{\mathrm{PR}}=2.202 \pm 0.001$ (see, e.g., Ref. [48]); one can use the Ginibre inequality [24] to conclude that the model(s) defined by Eq. (58) possess an ordering transition, whose transition temperature $\Theta_{\text {ord }}(A, B)$ is bounded by

$$
\min (A, B) \Theta_{\mathrm{PR}} \leq \Theta_{\mathrm{ord}}(A, B) \leq \max (A, B) \Theta_{\mathrm{PR}}
$$

On the other hand, when $0<B \ll A$, it is commonly believed that, above $\Theta_{\text {ord }}$ the system possess a disordered phase with slow decay of correlations and infinite susceptibility, i.e., a Berezinskiī-Kosterlitz-Thouless (BKT) phase, and, at higher temperature, a BKT transition [49-54] (we do not know any rigorous proof nor refutation of the conjecture); if this happens, then the following bound can be obtained, again by correlation inequalities

$$
\Theta_{\mathrm{BKT}}(A, B) \geq A \Theta_{\mathrm{BKT}}(d=2)
$$

where $\Theta_{\mathrm{BKT}}(d=2)$ denotes the transition temperature for the strict two-dimensional counterpart, whose numerical value is $\Theta_{\mathrm{BKT}}(d=2)=0.89 \pm 0.01$ [51]. On the other hand, according to Renormalization-Group treatments and in the same limit (see, e.g., Refs. [55,56])

$$
\begin{aligned}
\Theta_{\mathrm{BKT}}(A, B) & \approx A\left[\Theta_{B K T}(d=2)\right. \\
& \left.+(\pi / 2)^{2}|\log (B / A)|^{-2}\right] .
\end{aligned}
$$

\section{Comparison inequalities}

We consider here 3-component spins associated with a $d$-dimensional lattice and interacting via a ferromagnetic pair potential restricted to nearest neighbours and of the form

$$
U=U_{j k}=-\left(\sin \theta_{j} \sin \theta_{k}\right)^{l} \cos \left(\phi_{j}-\phi_{k}\right)
$$

where $l$ is an arbitrary positive integer; let $H_{\Lambda}$ denote the resulting Hamiltonian,

$$
H_{\Lambda}=-\sum_{\langle i j\rangle}\left(\sin \theta_{j} \sin \theta_{k}\right)^{l} \cos \left(\phi_{i}-\phi_{j}\right)
$$

let $Z_{\Lambda}$ denote the corresponding partition function, and let

$$
F_{p q}=\left\langle\left(\sin \theta_{p} \sin \theta_{q}\right)^{l} \cos \left(\phi_{p}-\phi_{q}\right)\right\rangle_{H_{\Lambda}}(\beta)
$$

denote the correlation function. Owing to the functional form of the integrands, and $\operatorname{since} \sin \theta=\sin (\pi-\theta)$, in the definition of $F_{p q}$, each integral over a variable $\theta_{j}$ ranging 
from 0 to $\pi$ is twice the integral of the same function of $\theta_{j}$ over the range between 0 and $\pi / 2$, and the powers of two resulting in both denominator and numerator cancel out identically; over the named range, $\sin \theta$ and $(\sin \theta)^{l}$ are monotonic increasing and invertible functions of the argument; thus, upon defining $r_{j}=\left(\sin \theta_{j}\right)^{l}$, single-site variables have the probability distribution

$$
\begin{aligned}
& d \mathcal{P}_{i}^{0} \propto d \mu\left(r_{i}\right) d \phi_{i} \\
& d \mu\left(r_{i}\right)=(1 / l) \frac{r_{i}^{(2 / l)}}{\sqrt{1-r_{i}^{(2 / l)}}} d r_{i} \\
& 0 \leq r_{i} \leq 1,0 \leq \phi_{i} \leq 2 \pi
\end{aligned}
$$

where the measure $d \mu\left(r_{j}\right)$ is not concentrated at 0 ; the thermal ensemble average (Eq. (65)) can be rewritten as as

$$
F_{p q}=\langle F(\varrho, \varphi)\rangle^{\chi}
$$

i. e., as expectation value with respect to the probability measure

$$
d \chi \propto \exp \left(-\beta H_{\Lambda}\right) \prod_{j} d \mathcal{P}_{j}^{0}
$$

Moreover, let $b$ denote a positive number, $0<b \leq 1$, and let $\langle F(\varrho, \varphi)\rangle_{b}$ denote the mean value obtained by constraining all $r$ variables to the value $b$, and let $Z_{\Lambda, b}$ denote the corresponding partition function; according to Well's inequality and its generalizations to continuous spins [25-29], there exists a positive number $a$ such that

$$
\langle F(\varrho, \varphi)\rangle_{1} \geq\langle F(\varrho, \varphi)\rangle^{\chi} \geq\langle F(\varrho, \varphi)\rangle_{a}
$$

By scaling, the potential model defined by Eq. (63) with $r_{j}=a, \forall j$ and at inverse temperature $\beta$ is the same as the one defined by $r_{j}=1, \forall j$ (i.e., planar rotators) at the inverse temperature $a^{2} \beta$.

Thus, when $d=2$ and $\forall l$, the potential model (63) produces disorder at all finite temperature and a BKTlike transition at low temperature; on the other hand, when $d=3$, the potential model supports an ordering transition at finite temperature.

The previous result can be generalized as follows: let now $s$ denote an arbitrary positive integer, and let us consider the mean value

$$
\begin{aligned}
\mathcal{F}_{j k, l s} & =\left\langle\left(\sin \theta_{j} \sin \theta_{k}\right)^{l s} \cos \left(\phi_{j}-\phi_{k}\right)\right\rangle_{H_{\Lambda}}(\beta) \\
& =\left(1 / Z_{\Lambda}\right) \int \prod_{x \in \Lambda} d \phi_{x} d \mu_{x}(\sin \theta) \exp \left(-\beta H_{\Lambda}\right)\left(\sin \theta_{j} \sin \theta_{k}\right)^{l s} \cos \left(\phi_{j}-\phi_{k}\right)
\end{aligned}
$$

(i) then it will be proven that $\exists a>0$ :

$$
\mathcal{F}_{j k, l s} \geq \mathcal{F}_{j k, l s ; a}=\left(1 / Z_{\Lambda, a}\right) \int \prod_{x \in \Lambda} \delta\left(r_{x}-a\right) d r_{x} d \phi_{x} \exp \left[\beta \sum_{i j}\left(r_{i} r_{j}\right) \cos \left(\phi_{i}-\phi_{j}\right)\right]\left(r_{j} r_{k}\right)^{s} \cos \left(\phi_{k}-\phi_{l}\right)
$$

where the above transformation $r_{j}=\left(\sin \theta_{j}\right)^{l}$ has been applied;

(ii) by the method of duplicated variables

$$
\begin{aligned}
\mathcal{F}_{j k, l s ; a} & =\prod_{x \in \Lambda} \int_{0}^{1} d \mu\left(\rho_{x}\right) \int_{0}^{1} d r_{x} \delta\left(r_{x}-a\right) \int_{0}^{2 \pi} d \phi_{x} \int_{0}^{2 \pi} d \phi_{x}^{\prime} \exp \left\{\beta \sum_{\langle i j\rangle}\left[\left(\rho_{i} \rho_{j}\right)+\left(r_{i} r_{j}\right)\right] \cos \left(\phi_{i}-\phi_{j}\right)\right\} \\
& \times\left(\left(\rho_{k} \rho_{m}\right)^{s}-\left(r_{k} r_{m}\right)^{s}\right) \cos \left(\phi_{k}-\phi_{m}\right) \geq 0
\end{aligned}
$$

moreover the trigonometric identity

$$
\cos \left(\phi_{k}-\phi_{m}\right)=\cos \phi_{k} \cos \phi_{m}+\sin \phi_{k} \sin \phi_{m}
$$

entails

$$
\int_{0}^{2 \pi} d \phi_{k} \cos \phi_{k} \int_{0}^{2 \pi} d \phi_{m} \cos \phi_{m} \geq 0
$$


morover, the defintions

$$
\rho_{k}=(1 / 2)\left[\left(\rho_{k}+r_{k}\right)+\left(\rho_{k}-r_{k}\right)\right], \quad r_{k}=(1 / 2)\left[\left(\rho_{k}+r_{k}\right)-\left(\rho_{k}-r_{k}\right)\right]
$$

imply

$$
\left(\rho_{k}-\rho_{m}\right)^{s}-\left(r_{k}-r_{m}\right)^{s}=\sum_{p, q, p^{\prime}, q^{\prime}} C_{p, q, p^{\prime}, q^{\prime}}\left(\rho_{k}+r_{k}\right)^{p}\left(\rho_{k}-r_{k}\right)^{q}\left(\rho_{m}+r_{m}\right)^{p^{\prime}}\left(\rho_{m}-r_{m}\right)^{q^{\prime}}
$$

where each coefficient $C_{p, q, p^{\prime}, q^{\prime}}$ is non-negative;

let us now consider

$$
\int_{0}^{1} d \mu(\rho) \int_{0}^{1} d r \delta(r-a)(\rho+r)^{I}(\rho-r)^{J}=\int_{0}^{1} d \mu(\rho)(\rho+a)^{J}\left(\rho_{a}\right)^{J}
$$

where $0<a<1$; if $J$ is even the integral is also positive; Since supp $\mu \neq\{0\}, \exists \epsilon>0$ : supp $\mu \cap[\epsilon, 1] \neq \emptyset$; $\mu$ is differentiable, and let $\nu=\frac{d \mu}{d \rho}$. Then $f_{I J}(a)=$ $\int_{0}^{1} d \rho \nu(\rho)(\rho+a)^{I}(\rho-a)^{J}$ is a continuous function $\epsilon$
$C[0,1]$, and $f_{I J}(a=0)=\int_{0}^{1} d \mu(\rho) \rho^{I+J}>0$; hence $\exists a^{*}, 0<a^{*}<1, \forall 0<a<a^{*}: f_{I J}(a)>0, \forall I, J$.

Therefore one obtains result $(i)$ for $a<a^{*}$ and arbitrary non-negative $l, s$.
[1] Ya. G. Sinaĩ, Theory of Phase Transitions; Rigorous Results, (Pergamon Press, Oxford, 1982).

[2] H.-O. Georgii, Gibbs Measures and Phase transitions, (de Gruyter, Berlin-New York, 1988).

[3] N. Angelescu, V. A. Zagrebnov, J. Phys. A 15, L639 (1982).

[4] N. Angelescu, S. Romano, V. A. Zagrebnov, Phys. Lett, A 200, 433 (1995).

[5] V. A. Zagrebnov, Physica A 232, 737 (1996).

[6] I. Dzyaloshinsky, J. Phys. Chem. Solids 4, 241 (1958); Zh. Eksp. Teor. Fiz. [Sov. Phys. - JETP] 10, 814 (1960).

[7] T. Moriya, Phys. Rev. Lett. 4, 228 (1960); Phys. Rev. 117, 635 (1960); 120, 91 (1960).

[8] T. Moriya, in Magnetism, a treatise on modern theory and materials, edited by G. T. Radom, H. Suhl (Academic Press, London and New York, 1963), Vol. 1, Chap. 3, p. 85-125.

[9] T. Nagamiya, Solid State Phys. 20, 306 (1967).

[10] D. D. Betts, in Phase Transitions and Critical Phenomena, (Academic Press, London and New York, 1974), Vol. 3, Chap. 8, p. 569-652.

[11] Y. R. Lin-Liu, Y. M. Shi, C.-W. Woo, H. T. Tan, Phys. Rev. A 14, 445 (1976).

[12] B. W. van der Meer, G. Vertogen, A. J. Dekker, J. G. J. Ypma, J. Chem. Phys. 65, 3935 (1976).

[13] B. W. van der Meer, G. Vertogen, in The Molecular Physics of Liquid Crystals, edited by G. R. Luckhurst, G. W. Gray, (Academic Press, London, 1979), Chap. 6, p. $149-168$.

[14] H. Schröder, in The Molecular Physics of Liquid Crystals, edited by G. R. Luckhurst, G. W. Gray, (Academic Press, London, 1979), Chap. 5, p. 121-147.

[15] L. Hu, Y. Jiang, R. Tao, Phys. Rev. E 57, 4289 (1998).
[16] J. Saha, B. Nandi, P. K. Mukherjee, M. Saha, Mol. Cryst. Liq. Cryst. 250, 185 (1994).

[17] G. R. Luckhurst, S. Romano, H. B. Zewdie, J. Chem. Soc. Faraday Trans. 92, 1781 (1996).

[18] R. Memmer, F. Janssen, Liq. Cryst. 24, 805 (1998).

[19] R. Memmer, F. Janssen, J. Chem. Soc. Faraday Trans. 94, 267 (1998).

[20] R. Memmer, F. Janssen, Z. Naturforsch. A 54, 747 (2000).

[21] Z. Zhidong, L. Jinwei, Z. Wu, Int. J. Mod. Phys. B 14, 475 (2000).

[22] G. A. Baker, Jr., Quantitative Theory of Critical Phenomena, (Academic Press, Boston, 1990).

[23] J. Glimm, A. Jaffe, Quantum Physics, a Functional Integral Point of View (Springer, Berlin-New York, 1981).

[24] J. Ginibre, Commun. Math. Phys. 16, 310 (1970).

[25] D. Wells, PhD Thesis, (Indiana University, 1977).

[26] J. Bricmont, J. L. Lebowitz, C.-E. Pfister, J. Stat. Phys. 24, 269 (1981).

[27] J. Bricmont, J. L. Lebowitz, C. E. Pfister, in The Wonderful World of Stochastics: A Tribute to Elliott W. Montroll, edited by M. F. Shlesinger, G. H. Weiss, (Elsevier, Amsterdam, 1985), Chap. 10, p. 206-213.

[28] J. Bricmont, J.-R. Fontaine, J. Stat. Phys. 26, 745 (1981).

[29] F. Dunlop, J. Stat. Phys. 41, 733 (1985).

[30] J. Fröhlich, Bull. Am. Math. Soc. 84, 165 (1978).

[31] J. Fröhlich, R. Israel, E. H. Lieb, B. Simon, Commun. Math. Phys. 62, 1 (1978).

[32] M. Campbell, L. Chayes, J. Phys. A 32, 8881 (1999).

[33] L. Chayes, S. B. Shlosman, V. A. Zagrebnov, J. Stat. Phys. 98, 537 (2000).

[34] H.-O. Carmesin, Phys. Lett. A 125, 294 (1987). 
[35] S. Romano, Nuovo Cimento B 100, 447 (1987).

[36] L. Klein, A. Aharony, Phys. Rev. B 44, 856 (1991).

[37] C. E. Cordeiro, E. V. de Mello, M. A. Continentino, Z. Phys. B 85, 307 (1993).

[38] A. R. Völkel, F. G. Mertens, A. R. Bishop, G. M. Wysin, Ann. Phys. (Leipzig) 2, 308 (1993).

[39] F. Lacerda, J. Ricardo da Sousa, I. P. Fittipaldi, J. Appl. Phys. 75, 5829 (1994).

[40] L. Biegala and J. Sznajd, Physica A 209, 422 (1994).

[41] C. E. Zaspel, Phys. Lett. A 94, 97 (1983).

[42] J. Curély, R. Georges, Phys. Lett. A 184, 310 (1994).

[43] J. Curély, Physica B 205, 31 (1995).

[44] A. B. Harris, R. D. Kamien, T. C. Lubensky, Phys. Rev. lett. 78, 1476 (1997).

[45] S. A. Issaenko, A. B. Harris, T. C. Lubensky, Phys. Rev. E 60, 578 (1999)

[46] S. A. Issaenko, A. B. Harris, Phys. Rev. E 61, 2777
(2000).

[47] A. V. Emelyanenko, M. A. Osipov, D. A. Dunmur, Phys. Rev. E 62, 2340 (2000)

[48] P. Butera, M. Comi, Phys. Rev. B 56, 8212 (1997).

[49] J. Fröhlich, T. Spencer, Commun. Math. Phys. 81, 527 (1981).

[50] P. Minnhagen, Rev. Mod. Phys. 59, 1001 (1987).

[51] R. Gupta, J. De Lapp, G. G. Batrouni, G. C. Fox, C. F. Baillie, J. Apostolakis, Phys. Rev. Lett. 61, 1996 (1988); R. Gupta, C. F. Baillie, Phys. Rev. B 45, 2883 (1992).

[52] P. Butera, M. Comi, Phys. Rev. B 50, 3052 (1994)

[53] S. W. Pierson, Philos. Mag. B 76, 715 (1997).

[54] Z. Gulácsi, M. Gulácsi, Adv. Phys. 47, 1 (1998).

[55] S. Hikami, T. Tsuneto, Prog. Theor. Phys. 63, 387 (1980).

[56] E. Rastelli, A. Tassi, J. Appl. Phys. 81, 4140 (1997).

\title{
ПРО СПІНОВІ МОДЕЛІ ІРАТКОВОГО ГАЗУ З КІРАЛЬНИМИ ВЗАЕМОДІЯМИ
}

\author{
С. Романо ${ }^{1}$, В. Загребнов ${ }^{2}$ \\ ${ }^{1}$ Національний інститут фізики матеріялів і фізичний факультет, \\ Університет м. Павія віа А. Бассі, I-27100, Павія, Італія \\ romano@pv.infn.it \\ ${ }^{2}$ Університет Середземного моря і Центр теоретичной фізики, \\ CNRS-Люміні 907, F-13288, Марсель, Франиіл \\ zagrebnov@cpt.univ-mrs.fr
}

На тривимірній гратщі вивчено моделі граткового газу, що складаються з двокомпонентних класичних спінів (одиничних векторів), які взаємодіють через парний потенщіял, що містить як скалярний добуток двох спінів, так і кіральний доданок. Таким моделям можна надати як магнетної, так і холестеричної інтерпретації. Доведено, що версія моделі, у якій усі вузли заповнені, переходить у впорядкований стан при скінченній температурі. Далі ми розглядаємо розведену версію моделі й доводимо існування порога хемічного потенціялу, понад яким модель дає далекий орієнтащійний порядок при достатньо низьких температурах. Також обговорено поширення цих строгих результатів на трикомпонентні спіни. 